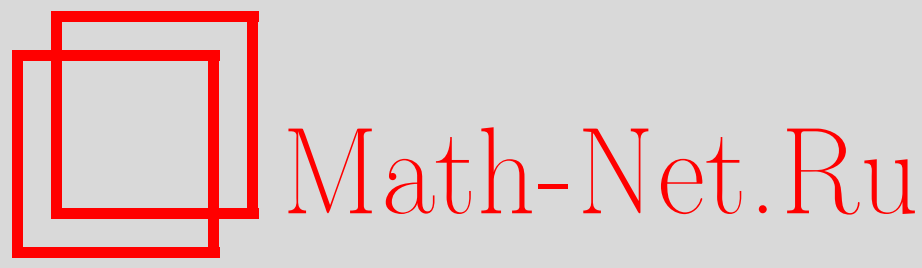

А. А. Гриб, Ю. В. Павлов, Физика высоких энергий в окрестности вращающихся черных дыр, ТМФ, 2015, том 185, номер 1, 77-85

DOI: https://doi.org/10.4213/tmf8910

Использование Общероссийского математического портала Math-Net.Ru подразумевает, что вы прочитали и согласны с пользовательским соглашением http://www . mathnet.ru/rus/agreement

Параметры загрузки:

IP : 54.92 .164 .108

26 апреля 2023 г., 05:42:20

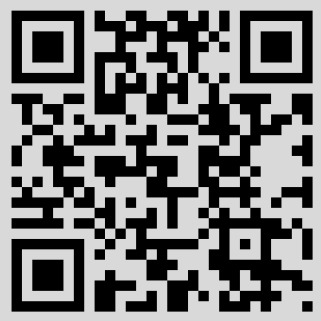




\title{
ФИЗИКА ВЫСОКИХ ЭНЕРГИЙ В ОКРЕСТНОСТИ ВРАЩАЮЩИХСЯ ЧЕРНЫХ ДЫР
}

\begin{abstract}
Рассмотрены столкновения частиц в окрестности горизонта вращающихся черных дыр. Показано, что существование геодезических, выходящих из-под гравитационного радиуса, как для массивных, так и для безмассовых частиц ведет к различным возможностям появления сколь угодно большой энергии столкновения в системе центра масс двух частиц. Дана классификация таких геодезических в общем случае на основе доказанной теоремы о свойствах экстремальных сферических орбит. Проанализированы случай роста энергии для ситуации, когда одна из частиц (критическая) движется по геодезической "белой дыры" и имеет близкий к предельному угловой момент, а другая падает по обычной геодезической, и случай сколь угодно большого отрицательного момента импульса первой частицы.
\end{abstract}

Ключевые слова: черные дыры, метрика Керра, столкновения частиц, геодезические.

DOI: $10.4213 / \operatorname{tmf} 8910$

\section{1. ВВЕДЕНИЕ}

В работе Банадоса, Силка, Веста [1] было показано существование в окрестности горизонта вращающейся черной дыры с экстремальным угловым моментом особого резонанса, приводящего к неограниченному росту энергии в системе центра масс движущихся частиц. Физическая причина появления такого резонанса проста. Одна из частиц, называемая критической, сильно "заворачивается" в эргосфере вращающейся черной дырой так, что радиальная компонента ее скорости близка к нулю. Другая (некритическая) частица имеет радиальную компоненту скорости, близкую к скорости света. Тем самым относительная скорость частиц стремится к скорости света, и неограниченно растущий лоренц-фактор ведет к неограниченному росту энергии в системе центра масс.

В наших работах [2]-[4] аналогичный эффект рассмотрен для неэкстремальных керровских черных дыр, поскольку есть мнение, что экстремальные черные дыры

* Российский государственный педагогический университет им. А. И. Герцена, СанктПетербург, Россия. E-mail: andrei_grib@mail.ru

†Лаборатория теоретической физики им. А. А. Фридмана, Санкт-Петербург, Россия

${ }^{\ddagger}$ Институт проблем машиноведения РАН, Санкт-Петербург, Россия.

E-mail: yuri.pavlov@mail.ru 
отсутствуют в природе в силу гравитационного излучения таких объектов [5]. Было показано, что критические частицы могут получаться в результате многократного столкновения, когда первоначально некритическая частица, прилетевшая к горизонту из внешнего пространства, после столкновения с также некритической частицей приводит к появлению критической частицы за счет передачи углового момента.

В наших работах [6], [7] было показано, что неограничено большие энергии в системе центра масс при столкновении двух частиц могут получаться в любой точке эргосферы, если одна из частиц обладает неограничено большим отрицательным угловым моментом. Однако не вполне ясно, как она может приобрести такой момент импульса.

Новым явлением, исследованным в статье [8], является роль особых геодезических, названных нами геодезическими "белой дыры", при рассмотрении столкновений частиц в эргосфере черной дыры. Как известно из учебников [9], вблизи горизонта черной дыры требование геодезической полноты требует существования, кроме обычных геодезических, приходящих из внешней по отношению к черной дыре области, геодезических, выходящих из-под гравитационного радиуса; их мы и назвали геодезическими “белой дыры". Такими, в частности, являются геодезические с отрицательной относительно бесконечности энергией [10], которые играют ключевую роль в механизме Пенроуза извлечения энергии из вращающейся черной дыры [11].

Настоящая работа является продолжением статьи [8]. В отличие от [8], где рассматриваются только экваториальные геодезические "белой дыры", доказана теорема, позволяющая дать классификацию таких линий в общем случае. Рассмотрены случаи движения не только массивных, но и безмассовых частиц. Затем нами изучается вопрос о неограниченном росте энергии столкновения в системе центра масс, когда одна из частиц движется по геодезической “белой дыры". Показано, что оба рассмотренных нами ранее эффекта могут иметь место без привлечения добавочных гипотез о приобретении критического углового момента или неограниченного роста абсолютной величины этого момента.

В статье используется система единиц, в которой гравитационная постоянная и скорость света равны единице: $G=c=1$.

\section{2. ГЕОДЕЗИЧЕСКИЕ “БЕЛОЙ ДЫРЫ” В МЕТРИКЕ КЕРРА}

Метрика Керра вращающейся черной дыры [12] в координатах Бойера-Линдквиста [13] имеет вид

$$
d s^{2}=d t^{2}-\frac{2 M r}{\rho^{2}}\left(d t-a \sin ^{2} \theta d \varphi\right)^{2}-\rho^{2}\left(\frac{d r^{2}}{\Delta}+d \theta^{2}\right)-\left(r^{2}+a^{2}\right) \sin ^{2} \theta d \varphi^{2}
$$

где

$$
\rho^{2}=r^{2}+a^{2} \cos ^{2} \theta, \quad \Delta=r^{2}-2 M r+a^{2}
$$

и $M$ - масса черной дыры, $a M-$ ее момент импульса; мы полагаем, что $0 \leqslant a \leqslant M$. Горизонт событий керровской черной дыры соответствует значению координаты

$$
r=r_{\mathrm{H}} \equiv M+\sqrt{M^{2}-a^{2}} .
$$

Поверхность предела статичности определяется значением

$$
r=r_{1} \equiv M+\sqrt{M^{2}-a^{2} \cos ^{2} \theta} .
$$


Область пространства-времени между пределом статичности и горизонтом событий называется эргосферой.

Уравнения геодезических в метрике Kерра (1) имеют вид (см. книги [14], § 62 или [15], § 3.4.1)

$$
\begin{gathered}
\rho^{2} \frac{d t}{d \lambda}=-a\left(a E \sin ^{2} \theta-J\right)+\frac{r^{2}+a^{2}}{\Delta} P, \\
\rho^{2} \frac{d \varphi}{d \lambda}=-\left(a E-\frac{J}{\sin ^{2} \theta}\right)+\frac{a P}{\Delta}, \\
\rho^{2} \frac{d r}{d \lambda}=\sigma_{r} \sqrt{R}, \quad \rho^{2} \frac{d \theta}{d \lambda}=\sigma_{\theta} \sqrt{\Theta}
\end{gathered}
$$

где

$$
\begin{gathered}
R=P^{2}-\Delta\left[m^{2} r^{2}+(J-a E)^{2}+Q\right], \quad P=\left(r^{2}+a^{2}\right) E-a J, \\
\Theta=Q-\cos ^{2} \theta\left[a^{2}\left(m^{2}-E^{2}\right)+\frac{J^{2}}{\sin ^{2} \theta}\right] .
\end{gathered}
$$

Здесь $E$ - энергия (относительно бесконечности) движущейся частицы, $E=$ const, $J$ - сохраняющаяся проекция ее момента импульса на ось вращения черной дыры, $m$ - масса покоя движущейся частицы. Для частицы с $m \neq 0$ параметр $\lambda=\tau / m$, где $\tau$ - ее собственное время; $Q$ - постоянная Картера, при движении в экваториальной плоскости $Q=0$. Постоянные $\sigma_{r}, \sigma_{\theta}= \pm 1$ определяют направление движения по координатам $r, \theta$.

Как следует из уравнений (6), параметры, характеризующие любую геодезическую, должны удовлетворять условиям

$$
R \geqslant 0, \quad \Theta \geqslant 0 .
$$

Для геодезической, соответствующей траектории движущейся пробной частицы снаружи горизонта событий, должно выполняться также условие движения “вперед во времени"

$$
\frac{d t}{d \lambda} \geqslant 0
$$

Условия (9), (10) приводят к следующим неравенствам для возможных значений энергии $E$ и проекции углового момента $J$ пробной частицы в точке с координатами $(r, \theta)$ при фиксированном значении $\Theta \geqslant 0[7]$ :

- снаружи эргосферы, в области $r^{2}-2 r M+a^{2} \cos ^{2} \theta>0$,

$$
\begin{gathered}
E \geqslant \frac{1}{\rho^{2}} \sqrt{\left(m^{2} \rho^{2}+\Theta\right)\left(r^{2}-2 r M+a^{2} \cos ^{2} \theta\right)}, \\
J_{-}(r, \theta) \leqslant J \leqslant J_{+}(r, \theta),
\end{gathered}
$$

где

$$
\begin{aligned}
J_{ \pm}(r, \theta)= & \frac{\sin \theta}{r^{2}-2 r M+a^{2} \cos ^{2} \theta}[-2 r M a E \sin \theta \pm \\
& \left. \pm \sqrt{\Delta\left(\rho^{4} E^{2}-\left(m^{2} \rho^{2}+\Theta\right)\left(r^{2}-2 r M+a^{2} \cos ^{2} \theta\right)\right)}\right]
\end{aligned}
$$

- внутри эргосферы, в области $r_{\mathrm{H}}<r<r_{1}(\theta), r^{2}-2 r M+a^{2} \cos ^{2} \theta<0$,

$$
J \leqslant J_{+}(r, \theta) \leqslant J_{-}(r, \theta),
$$


а значение энергии частицы может быть любым, как положительным, так и отри-

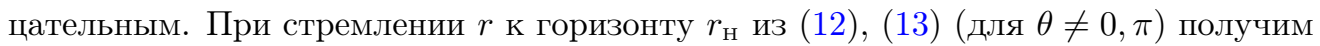

$$
J \leqslant J_{\mathrm{H}}=\frac{2 M r_{\mathrm{H}} E}{a} .
$$

Следовательно, $J_{\text {н }}$ является верхней границей для проекции момента импульса частицы с энергией $E$ у горизонта событий черной дыры.

Ранее в работе [10] было показано, что геодезические с отрицательной относительно бесконечности энергией в эргосфере начинаются и заканчиваются при $r=r_{\text {н }}$. Существуют ли геодезические с положительной энергией, которые снаружи горизонта событий начинаются и заканчиваются при значении $r=r_{\mathrm{H}}$ ? Исследуем этот вопрос.

Введем эффективный потенциал для радиального движения

$$
V_{\text {eff }}=-\frac{R}{2 \rho^{4}} \text {. }
$$

Тогда вследствие (6) имеем

$$
\frac{1}{2}\left(\frac{d r}{d \lambda}\right)^{2}+V_{\mathrm{eff}}=0, \quad \frac{d^{2} r}{d \lambda^{2}}=-\frac{\partial V_{\mathrm{eff}}}{\partial r} .
$$

Для ответа на поставленный выше вопрос достаточно найти области значений энергии частиц, при которых

$$
r>r_{\mathrm{H}}, \quad V_{\mathrm{eff}}(r)=0 \quad \Longrightarrow \quad \frac{\partial V_{\mathrm{eff}}}{\partial r}>0 .
$$

Действительно, требование (17) означает, что в верхней по $r$ точке траектории ускорение $d^{2} r / d \lambda^{2}$ в соответствии с (16) направлено в сторону уменьшения радиальной координаты, т. е. к горизонту событий. Для нахождения граничных значений параметров для таких геодезических “белой дыры" рассмотрим систему уравнений

$$
V_{\text {eff }}(r)=0, \quad V_{r}^{\prime} \equiv \frac{\partial V_{\text {eff }}}{\partial r}=0
$$

Эта система уравнений определяет орбиты с постоянным значением радиальной координаты, так называемые сферические орбиты [16].

Для светоподобных геодезических $(m=0)$ система уравнений $(18)$ приводит к выражениям

$$
\frac{J}{E}=\frac{r^{2}(3 M-r)-a^{2}(r+M)}{a(r-M)}, \quad \frac{a^{2} Q}{E^{2}}=\frac{r^{3}\left(4 M a^{2}-r(r-3 M)^{2}\right)}{(r-M)^{2}} .
$$

Для невращающейся черной дыры $(a=0)$ сферические фотонные орбиты будут круговыми со значениями

$$
J= \pm 3 \sqrt{3} M E, \quad r=3 M .
$$

В области $2 M<r<3 M$ внутри радиуса фотонной круговой орбиты метрики Шварцшильда все светоподобные геодезические с $|J| / M E>3 \sqrt{3}$ начинаются и заканчиваются на гравитационном радиусе $r_{\mathrm{H}}=2 M$.

Для вращающейся черной дыры Керра радиусы экваториальных фотонных круговых орбит $Q=0$ могут быть найдены из соотношений (19) как корни уравнения $r(r-3 M)^{2}-4 M a^{2}=0$, и снаружи горизонта событий они равны [17]

$$
r_{ \pm}=2 M\left[1+\cos \left(\frac{2}{3} \arccos \frac{\mp a}{M}\right)\right] .
$$




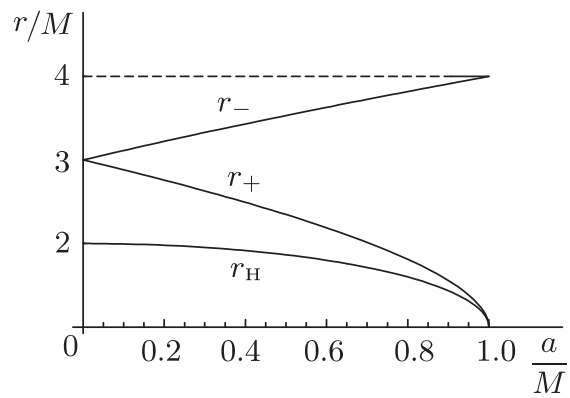

a

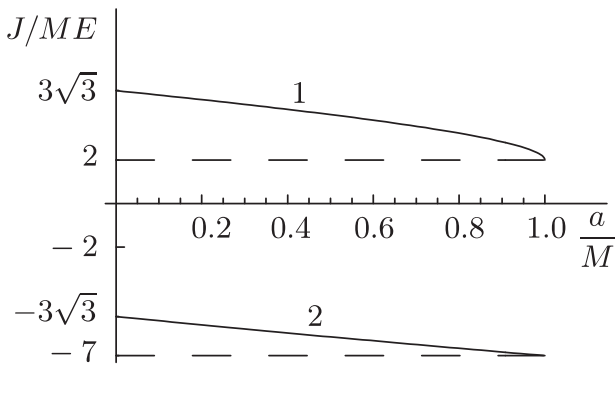

6

Рис. 1. Радиусы (а) и моменты импульса (б) круговых экваториальных фотонных орбит в метрике Керра.

Значения $r_{ \pm}$и отвечающие им отношения $J / M E$ представлены на рис. 1.

Можно убедиться, что полученные значения $J / M E$ совпадают с предельными величинами $J_{ \pm}\left(r_{ \pm}, \pi / 2\right) / M E$, вычисленными по формулам (12). Поэтому, если в области $r_{\mathrm{H}}<r<r_{+}$значения $J / M E$ превышают величины, соответствующие кривой 1 на рис. 1б, такие геодезические начинаются и заканчиваются на гравитационном радиусе $r_{\mathrm{H}}$. Если в области $r_{\mathrm{H}}<r<r_{-}$значения $J / M E$ меньше величины, соответствующей кривой 2 на рис. 1б, то такие геодезические начинаются и заканчиваются на гравитационном радиусе $r_{\mathrm{H}}$. Отметим, что в соответствии с неравенством (14) у горизонта событий $J / M E<2 r_{\mathrm{H}} / a$.

\section{3. О СВОЙСТВАХ СФЕРИЧЕСКИХ ОРБИТ В МЕТРИКЕ КЕРРА}

Как показано выше, вопрос о граничных значениях параметров, при которых геодезические снаружи горизонта событий начинаются и заканчиваются при $r=r_{\mathrm{H}}$, сводится к поиску граничных значений таких параметров для сферических орбит. В случае $m \neq 0$ массивных частиц минимальное значение энергии для сферической орбиты ограничивает максимально возможную энергию для таких геодезических "белой дыры".

Система уравнений (18), определяющая сферические орбиты, с учетом определения (15) может быть записана в виде

$$
R(r, m, E, J, Q)=0, \quad \frac{\partial R(r, m, E, J, Q)}{\partial r}=0 .
$$

Поэтому зависимость параметров сферических орбит от координаты $\theta$, которая имеется в эффективном потенциале, отсутствует.

При фиксированном $m \neq 0$ условия (22) определяют (возможно, неоднозначно) значения энергии и проекции момента импульса как функции радиальной координаты $r$ и константы Картера $Q$ :

$$
E=E(r, Q), \quad J=J(r, Q) .
$$

Дифференцируя равенства (18) по $r$, с учетом (23) получим

$$
\begin{gathered}
\frac{\partial V}{\partial r}+\frac{\partial V}{\partial E} \frac{\partial E}{\partial r}+\frac{\partial V}{\partial J} \frac{\partial J}{\partial r}=0, \\
\frac{\partial^{2} V}{\partial r^{2}}+\frac{\partial V_{r}^{\prime}}{\partial E} \frac{\partial E}{\partial r}+\frac{\partial V_{r}^{\prime}}{\partial J} \frac{\partial J}{\partial r}=0 .
\end{gathered}
$$


Если требуется найти экстремальную по моменту импульса сферическую орбиту при фиксированных значениях $Q$, т. е. решить уравнение $\partial J / \partial r=0$, то из (24) получим

$$
V=0, \quad V_{r}^{\prime}=0, \quad \frac{\partial J(r, Q)}{\partial r}=0 \quad \Longrightarrow \quad \frac{\partial V}{\partial E} \frac{\partial E}{\partial r}=0 .
$$

Можно показать, что для любых пробных частиц в метрике Керра

$$
V=0, \quad V_{r}^{\prime}=0, \quad \frac{\partial J(r, Q)}{\partial r}=0 \quad \Longrightarrow \quad \frac{\partial V}{\partial E} \neq 0 .
$$

Таким образом, экстремальная по моменту импульса сферическая орбита является также экстремальной по значению энергии: $\partial E / \partial r=0$.

Если требуется найти экстремальную по энергии сферическую орбиту при фиксированных значениях $Q$, т. е. решить уравнение $\partial E / \partial r=0$, то из $(24)$ получим

$$
V=0, \quad V_{r}^{\prime}=0, \quad \frac{\partial E(r, Q)}{\partial r}=0 \quad \Longrightarrow \quad \frac{\partial V}{\partial J} \frac{\partial J}{\partial r}=0 .
$$

Элементарные, но громоздкие вычисления показывают, что из уравнений

$$
V=0, \quad V_{r}^{\prime}=0, \quad \frac{\partial E(r, Q)}{\partial r}=0, \quad \frac{\partial V}{\partial J}=0
$$

мы имеем

$$
a \neq 0, \quad m \neq 0, \quad r=6 M, \quad E=\frac{2 \sqrt{2}}{3} m, \quad J=-\frac{\sqrt{2}}{3 m a}, \quad Q=12 m^{2} M^{2} .
$$

За этим исключением экстремальная по энергии сферическая орбита является также экстремальной по проекции момента импульса: $\partial J / \partial r=0$.

Из уравнения (25) получаем

$$
V=0, \quad V_{r}^{\prime}=0, \quad \frac{\partial E}{\partial r}=0, \quad \frac{\partial J}{\partial r}=0 \quad \Longrightarrow \quad \frac{\partial^{2} V}{\partial r^{2}}=0 .
$$

Если при фиксированных параметрах геодезической $(m, E, J, Q)$ и при малом отклонении от значения $r$, соответствующего сферической орбите, возникает сила $d^{2} r / d \lambda^{2}$, стремящаяся вернуть значение $r$ к первоначальной величине, то такие сферические орбиты называют устойчивыми. Из равенств (16) и (18) следует, что сферическая орбита устойчива, если

$$
V_{\mathrm{eff}}(r)=0, \quad \frac{\partial V_{\mathrm{eff}}}{\partial r}=0, \quad \frac{\partial^{2} V_{\mathrm{eff}}}{\partial r^{2}}>0 .
$$

Таким образом, вследствие (30) все экстремальные по проекции момента импульса или экстремальные по энергии (за исключением случая (29)) сферические орбиты являются также предельными устойчивыми сферическими орбитами.

В результате нами доказана следующая теорема об экстремальных орбитах.

ТЕОрема. Сферическая орбита в поле черной дыры Керра, экстремальная по значению проекции момента импульса частицы (экстремальная по энергии, за исключением случая (29)), при фиксированной константе Картера является также экстремальной по значению энергии (по значению проекиии момента импульса) и предельной устойчивой сферической орбитой.

При $Q=0$ и $\theta=\pi / 2$ получаем очевидное следствие. 
СлеДСТвИЕ. Круговая экваториальная орбита в метрике Керра, экстремальная по значению энергии (момента импульса), является также экстремальной по значению момента импульса (энергии) и предельной устойчивой круговой орбиmoü.

Для ньютоновского закона тяготения уравнения (23) в обычной системе единиц принимают вид

$$
E=m c^{2}-G \frac{m M}{2 r}, \quad J= \pm m \sqrt{G M r} .
$$

Все круговые орбиты являются устойчивыми. Экстремальные значения по энергии и моменту импульса имеют место лишь на границах, при $r \rightarrow \infty$ или $r \rightarrow 0$.

Случай метрики Шварцшильда разобран нами в статье [8], где показано, что при энергиях частиц $E<m \cdot 2 \sqrt{2} / 3$ геодезические снаружи горизонта событий черной дыры начинаются и заканчиваются на гравитационном радиусе $r=2 M$.

При решении системы уравнений (18) для метрики Керра после элементарных, но громоздких алгебраических преобразований получим

$$
\varepsilon \equiv \frac{E}{m}=\frac{x^{3}(x-2)+A H_{ \pm}}{x^{2} \sqrt{x^{3}(x-3)+2 A H_{ \pm}}}, \quad l \equiv \frac{J}{m M}=\frac{\left(x^{2}+A^{2}\right) H_{ \pm}-2 A x^{3}}{x^{2} \sqrt{x^{3}(x-3)+2 A H_{ \pm}}},
$$

где

$$
x=\frac{r}{M}, \quad A=\frac{a}{M}, \quad q=\frac{Q}{m^{2} M^{2}}, \quad H_{ \pm}= \pm \sqrt{x^{5}+A^{2} q^{2}+q x^{3}(3-x)}-A q .
$$

При $Q=0$ формулы (33) переходят в известные соотношения для экваториальных круговых орбит метрики Керра [17]. Результаты, полученные для этого случая в работе [8] из анализа формул (33), прямо следуют из доказанной выше теоремы и известных выражений для параметров предельных круговых устойчивых орбит. В частности, если энергия предельной устойчивой орбиты черной дыры с $a=M$ равна $E=m / \sqrt{3}$, то все экваториальные геодезические с энергией $E<m / \sqrt{3}$ снаружи горизонта начинаются и заканчиваются при $r=r_{\mathrm{H}}$.

Дифференцируя первое из уравнений (33), получим

$$
\frac{\partial \varepsilon}{\partial q}=\frac{ \pm A\left(x^{3}-A H_{ \pm}\right)}{2 x^{2} \sqrt{x^{3}(x-3)+2 A H_{ \pm}} \sqrt{x^{5}+A^{2} q^{2}+q x^{3}(3-x)}} .
$$

Для произвольных геодезических с $E<m$ константа Картера $Q \geqslant 0$ в силу соотношений (6), (8). В этом случае положительные значения $J$ для сферических орбит возможны только при выборе $H_{+}$в (33). Тогда из (35) получим, что $\partial \varepsilon / \partial q>0$ при $r>r_{\text {н }}$ и, следовательно, для экстремальных $(\partial \varepsilon / \partial x=0)$ сферических орбит справедливо неравенство

$$
\frac{d \varepsilon}{d q}=\frac{\partial \varepsilon}{\partial x} \frac{d \varepsilon}{\partial q}+\frac{\partial \varepsilon}{\partial q}=\frac{\partial \varepsilon}{\partial q}>0
$$

На границах области существования сферических орбит с $H_{+}>0$ при $r>r_{\mathrm{H}}$ можно проверить, что энергия не превышает соответствующих значений для экваториальных круговых орбит. Поэтому предельные значения энергий массивных частиц, найденные для экваториальных геодезических в работе [8], сохраняются и при неэкваториальном движении с $J>0$, в частности, все геодезические с энергией $E<m / \sqrt{3}$ и $J>0$ снаружи горизонта керровской черной дыры с $a=M$ начинаются и заканчиваются при $r=r_{\mathrm{H}}$. 


\section{4. ЭНЕРГИЯ СТОЛКНОВЕНИЯ ЧАСТИЦ В СИСТЕМЕ ЦЕНТРА МАСС}

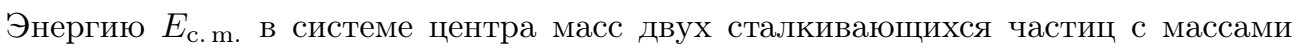
покоя $m_{1}$ и $m_{2}$ найдем, возведя в квадрат вектор

$$
\left(E_{\text {c. m. }}, 0,0,0\right)=p_{(1)}^{i}+p_{(2)}^{i},
$$

где $p_{(n)}^{i}-4$-импульсы частиц $(n=1,2)$. Возводя выражение $(37)$ в квадрат и учитывая уравнения $(5),(6)$ и $p_{(n)}^{i} p_{(n) i}=m_{n}^{2}$, получим [18]

$$
\begin{aligned}
E_{\mathrm{c} . \mathrm{m} .}^{2}= & m_{1}^{2}+m_{2}^{2}+\frac{2}{\rho^{2}}\left[\frac{P_{1} P_{2}-\sigma_{1 r} \sqrt{R_{1}} \sigma_{2 r} \sqrt{R_{2}}}{\Delta}-\right. \\
& \left.-\frac{\left(J_{1}-a E_{1} \sin ^{2} \theta\right)\left(J_{2}-a E_{2} \sin ^{2} \theta\right)}{\sin ^{2} \theta}-\sigma_{1 \theta} \sqrt{\Theta_{1}} \sigma_{2 \theta} \sqrt{\Theta_{2}}\right] .
\end{aligned}
$$

Найдем предел этого выражения при $r \rightarrow r_{\mathrm{H}}$, получим для падающих $\left(\sigma_{1 r}=\sigma_{2 r}=1\right)$ в экваториальной плоскости $(\theta=\pi / 2)$ частиц [19] следующее равенство:

$$
\begin{aligned}
\left.E_{\mathrm{c.m} .}^{2}(r)\right|_{r \rightarrow r_{\mathrm{H}}}= & \frac{\left(J_{1 H} J_{2}-J_{2 H} J_{1}\right)^{2}}{4 M^{2}\left(J_{1 H}-J_{1}\right)\left(J_{2 H}-J_{2}\right)}+ \\
& +m_{1}^{2}\left[1+\frac{J_{2 H}-J_{2}}{J_{1 H}-J_{1}}\right]+m_{2}^{2}\left[1+\frac{J_{1 H}-J_{1}}{J_{2 H}-J_{2}}\right],
\end{aligned}
$$

Поэтому энергия столкновения в системе центра масс неограниченно возрастает, если $r \rightarrow r_{\mathrm{H}}$ и момент импульса при фиксированной относительно бесконечности энергии одной из частиц (критической) стремится к предельно допустимому у горизонта событий значению $J_{\mathrm{H}}$ (см. условие (14)). Однако, за исключением случая предельно вращающейся черной дыры, когда $a=M$, частицы с моментом импульса $J_{\mathrm{H}}$ не могут, падая из бесконечности, достичь горизонта. Эффективная потенциальная энергия создает барьер, и большие значения энергии столкновений в системе центра масс могут быть достигнуты лишь при распадах или промежуточных столкновениях [2], [3], когда происходит увеличение момента импульса одной из падающих частиц и $J \rightarrow J_{\mathrm{H}}$. Другая возможность неограниченного возрастания энергии столкновений на горизонте событий - встречные столкновения, например, с частицами, движущимися по геодезическим “белых дыр" [8]. Однако, как легко увидеть, скажем, из диаграммы Пенроуза для вечных черных дыр, такие столкновения при $r \rightarrow r_{\mathrm{H}}$ могли бы осуществляться только в бесконечно далеком прошлом.

Здесь мы отметим другую возможность осуществления столкновений с неограниченно большой энергией. Из соотношения (38) получим, что в эргосфере в пределе $J_{2} \rightarrow-\infty$

$$
E_{\mathrm{c} . \mathrm{m} .}^{2} \approx J_{2} \frac{r^{2}-2 r M+a^{2} \cos ^{2} \theta}{\rho^{2} \Delta \sin ^{2} \theta}\left(\sigma_{1 r} \sqrt{J_{1+}-J_{1}}-\sigma_{2 r} \sqrt{J_{1-}-J_{1}}\right)^{2} \rightarrow+\infty .
$$

Энергия столкновения неограниченно возрастает (при фиксированной энергии частиц относительно бесконечности), когда момент импульса одной из частиц в эргосфере стремится к $-\infty$. Поскольку из бесконечности частица с $J_{2} \rightarrow-\infty$ не может попасть в эргосферу, для реализации этого условия необходим либо механизм промежуточных столкновений [6], [7], либо наличие сильных магнитных полей специального вида. 
Однако если столкновение падающей из бесконечности частицы происходит с частицей, движущейся в эргосфере по геодезической “белой дыры”, для которой согласно (13) нет ограничений снизу на проекцию момента импульса, то энергия столкновения в системе центра масс может быть сразу сколь угодно велика, причем в любой точке эргосферы.

Для геодезических “белых дыр" вблизи горизонта верхняя граница допустимого момента импульса частиц стремится к $J_{\mathrm{H}}$ (см. формулу (14)). При столкновении у горизонта с падающей из бесконечности частицей с таким моментом импульса, движущейся по геодезической “белой дыры”, энергия столкновения (39) также будет велика. Необходимость в промежуточных столкновениях при этом отпадает.

Благодарности. Работа поддержана РФФИ (грант № 15-02-06818_a).

\section{Список литературы}

[1] M. Bañados, J. Silk, S. M. West, Phys. Rev. Lett., 103:11 (2009), 111102, 4 pp.

[2] A. A. Grib, Yu. V. Pavlov, Писъма в ЖКЭТФ, 92:3 (2010), 147-151.

[3] A. A. Grib, Yu. V. Pavlov, Astropart. Phys., 34:7 (2011), 581-586, arXiv: 1001.0756.

[4] A. A. Grib, Yu. V. Pavlov, Grav. Cosmol., 17:1 (2011), 42-46, arXiv: 1010.2052.

[5] K.S. Thorne, Astrophys. J., 191 (1974), 507-519.

[6] А. А. Гриб, Ю. В. Павлов, ТМФ, 176:1 (2013), 60-68.

[7] A. A. Grib, Yu. V. Pavlov, Europhys. Lett., 101:2 (2013), 20004, 4 pp.

[8] A. A. Grib, Yu. V. Pavlov, Grav. Cosmol., 21:1 (2015), 13-18, arXiv: 1410.5736.

[9] Л. Д. Ландау, Е. М. Лифшиц, Теория поля, Наука, М., 1988.

[10] A. A. Grib, Yu. V. Pavlov, V. D. Vertogradov, Modern Phys. Lett. A, 29:20 (2014), 1450110, 9 pp., arXiv: 1304.7360.

[11] R. Penrose, Rivista Nuovo Cimento, 1 (1969), 252-276.

[12] R. P. Kerr, Phys. Rev. Lett., 11:5 (1963), 237-238.

[13] R. H. Boyer, R. W. Lindquist, J. Math. Phys., 8:2 (1967), 265-281.

[14] С. Чандрасекар, Математическая теория черных дыр, Мир, М., 1986.

[15] И. Д. Новиков, В. П. Фролов, Физика черных дыр, Наука, М., 1986.

[16] И. Г. Дымникова, УФН, 148:3 (1986), 393-432.

[17] J. M. Bardeen, W. H. Press, S. A. Teukolsky, Astrophys. J., 178 (1972), 347-369.

[18] T. Harada, M. Kimura, Phys. Rev. D, 83:8 (2011), 084041, 9 pp., arXiv: 1102.3316.

[19] A. A. Grib, Yu. V. Pavlov, O.F. Piattella, Grav. Cosmol., 18:1 (2012), 70-75, arXiv: 1203.4952 . 